\title{
Synchronization and Communication Using Semiconductor Lasers With Optoelectronic Feedback
}

\author{
Henry D. I. Abarbanel, Matthew B. Kennel, Lucas Illing, S. Tang, H. F. Chen, and J. M. Liu
}

\begin{abstract}
Semiconductor lasers provide an excellent opportunity for communication using chaotic waveforms. We discuss the characteristics and the synchronization of two semiconductor lasers with optoelectronic feedback. The systems exhibit broadband chaotic intensity oscillations whose dynamical dimension generally increases with the time delay in the feedback loop. We explore the robustness of this synchronization with parameter mismatch in the lasers, with mismatch in the optoelectronic feedback delay, and with the strength of the coupling between the systems. Synchronization is robust to mismatches between the intrinsic parameters of the lasers, but it is sensitive to mismatches of the time delay in the transmitter and receiver feedback loops. An open-loop receiver configuration is suggested, eliminating feedback delay mismatch issues. Communication strategies for arbitrary amplitude of modulation onto the chaotic signals are discussed, and the bit-error rate for one such scheme is evaluated as a function of noise in the optical channel.
\end{abstract}

Index Terms-Chaos, communication system nonlinearities, optical communication, optoelectronic devices, synchronization.

\section{INTRODUCTION}

$\mathbf{T}$ HE USE of chaotic waveforms for optical communication has been widely investigated in both theoretical and experimental contexts [1]-[3]. The use of chaotic signals on which to modulate and demodulate information may be quite attractive from the point of view of the efficiency of the use of communications channel bandwidth or possibly for reasons of power efficiency in the design and use of the transmitter. Issues of "security" of chaotic transmissions are a much more difficult topic, and little real insight has been presented in the literature on this matter. We do not consider this issue here.

Optical communications using chaotic transmitters and receivers offers the potential of an enormous usable bandwidth for high bit rate data communication or utilization of the available bandwidth by many users. Our earlier work on optical chaotic

Manuscript received February 5, 2001; revised June 18, 2001. This work was supported in part by the U.S. Department of Energy, Office of Basic Energy Sciences, Division of Engineering and Geosciences, under Grant DE-FG0390ER14138, by the National Science Foundation under Grant NCR-9612250, and by the U.S. Army Research Office under Contract DAAG55-98-1-0269.

H. D. I. Abarbanel is with the Institute for Nonlinear Science, Department of Physics and Marine Physical Laboratory, Scripps Institution of Oceanography, University of California, La Jolla, CA 92093-0402 USA (e-mail: hdia@jacobi.ucsd.edu).

M. B. Kennel is with the Institute for Nonlinear Science, University of California at San Diego, La Jolla, CA 93093-0402 USA (e-mail: mkennel@ucsd.edu).

L. Illing is with the Department of Physics and Institute for Nonlinear Science, University of California, San Diego, La Jolla, CA 93093-0402 USA (e-mail: illing@dirac.ucsd.edu).

S. Tang, H. F. Chen, and J. M. Liu are with the Department of Electrical Engineering, University of California at Los Angeles, Los Angeles, CA 90095159410 USA.

Publisher Item Identifier S 0018-9197(01)08343-9. communication, both theoretical and experimental, studied ring laser systems in which the active element was a section of rare earth doped fiber [4]. In the case of erbium-doped fiber, one can achieve substantial amplification at the wavelength of $1.55 \mu \mathrm{m}$, which is at a minimum of attenuation in standard single-mode optical fibers. The demonstration that such ring lasers could be used for communications at observed bit rates up to $250 \mathrm{Mb} / \mathrm{s}$ is quite important, in principle [3], but the fact that the upper lasing level in erbium has a lifetime of about $10 \mathrm{~ms}$ means that the chaotic waveform circulating in the ring changes very slowly on the time scale of the round-trip time of a standard ring laser composed of tens of meters of passive fiber. The ratio of fluorescent lifetime to laser ring round-trip time ratio is about $10^{5}$, and once the erbium upper level is populated, that population remains essentially constant and drops out of the dynamics of the ring laser. If there were no nonlinear effects in the ring, this laser would not exhibit chaotic oscillations. The observed chaotic waveforms then arise from the nonlinear effects of the glass fiber which, small in magnitude, act on the circulating light many times.

This circumstance has led us to consider an active element in a feedback laser system which has time scales commensurate with bit rates of gigabits per second that would be attractive for any realistic applications. We explore here the use of a semiconductor laser as the active element in a delay-feedback ring system. Except for the feedback-delay time, the carrier lifetime, the photon lifetime, and other time scales in this system are 1 ns or less, and the bandwidth for communications available to a chaotic signal is a few gigahertz or more. From the point of view of dynamics, the use of a semiconductor laser as the active element for a delay-feedback ring is also much more interesting, as the carrier inversion does not "freeze out" from the dynamical equations. Chaotic oscillations are achieved at gigahertz frequencies.

In this paper, we consider a laser system with feedback formed by an optoelectronic loop from the semiconductor laser optical output back to the bias current across the laser itself. This particular form of feedback is insensitive to the optical phase of the laser output. As intensity detection is much easier than phase detection, removing it from our dynamical considerations makes any strategy for communications much more realistic.

\section{SEMiconductor FeEdBack LASER}

\section{A. Equations of Motion}

We consider a closed-loop optical system, as seen in Fig. 1. The semiconductor laser is driven by a dc bias current $J_{0}$, and 


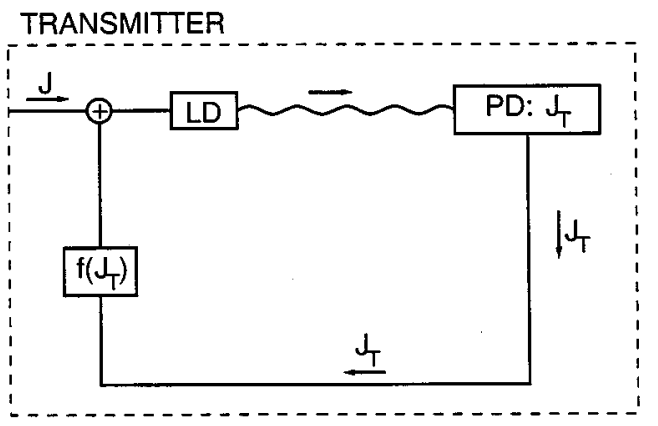

Fig. 1. Schematic of chaotic transmitter laser. The light from the semiconductor laser $(L D)$ is received by the photodiode $(P D)$ which puts out a current $J(t)$. (The symbol $P D: J(t)$ is shorthand for this.) This current is transformed by a nonlinear function into the current $J_{F}(t)=f\left(J\left(t-\tau_{R}\right)\right)$, which acts after a length of feedback loop $c \tau_{R}$ has been traversed. This current $\left(J_{F}(t)\right)$ is added to the external bias current $J_{0}$ and fed back into the semiconductor laser.

lases in a single mode of optical frequency $\omega_{0}$ whose complex valued coefficient is $A(t)$. The output light is received by a photodetector (PD) producing a current $J_{T}(t)$ proportional to $|A(t)|^{2}$. We also consider the possibility of placing a nonlinear function of the intensity $f\left(J_{T}(t)\right)$ in the electronic feedback loop from the PD back to the laser yielding a feedback current $J_{F}(t)=f\left(J_{T}\left(t-\tau_{R}\right)\right)$ with $\tau_{R}$ the time delay associated with the electronic feedback loop. The dynamical equations for $A(t)$ and the carrier density $N(t)$ are

$$
\begin{aligned}
\frac{d A(t)}{d t}= & -\frac{\gamma_{\mathrm{c}}}{2} A(t)+i\left(\omega_{0}-\omega_{\mathrm{c}}\right) A(t) \\
& +\frac{\Gamma}{2}(1-i b) g\left(N,|A|^{2}\right) A(t)+F_{\mathrm{sp}} \\
\frac{d N(t)}{d t}= & \frac{J_{0}+J_{F}(t)}{e d}-\gamma_{\mathrm{s}} N(t)-\frac{2 \epsilon_{0} n^{\prime 2}}{\hbar \omega_{0}} g\left(N,|A|^{2}\right)|A(t)|^{2} \\
= & \frac{J_{0}+f\left(J\left(t-\tau_{R}\right)\right)}{e d}-\gamma_{\mathrm{s}} N(t)-\frac{2 \epsilon_{0} n^{\prime 2}}{\hbar \omega_{0}} g\left(N,|A|^{2}\right)|A(t)|^{2} .
\end{aligned}
$$

where

$\begin{array}{ll}\gamma_{c} & \text { cavity decay rate; } \\ \omega_{0} & \text { center optical frequency; } \\ \omega_{\mathrm{c}} & \text { longitudinal mode frequency of the cold } \\ & \text { laser cavity; } \\ \Gamma & \text { confinement factor; } \\ b & \text { linewidth enhancement factor; } \\ g\left(N(t),|A(t)|^{2}\right) & \begin{array}{l}\text { optical gain coefficient including non- } \\ \text { linear effects; }\end{array} \\ d & \text { active layer thickness of the laser; } \\ n^{\prime} & \text { refractive index of the semiconductor } \\ & \text { medium; } \\ \gamma_{\mathrm{s}} & \text { spontaneous carrier decay rate; } \\ F_{\mathrm{sp}}=F_{R}+i F_{I} & \text { spontaneous emission noise source. }\end{array}$

We take this noise to be a Gaussian Langevin source with correlations $\left\langle F_{R}(t) F_{R}(s)\right\rangle=\left\langle F_{I}(t) F_{I}(s)\right\rangle=\left(R_{\mathrm{sp}} / 2\right) \delta(t-s)$ and $\left\langle F_{R}(t) F_{I}(s)\right\rangle=0$.

Ignoring the noise, the phase of the optical field is determined by $N(t)$ and $|A(t)|^{2}$ and is not an independent dynamical variable, so chaotic oscillations cannot appear without a nontrivial feedback $J_{F}(t)$, because chaos cannot occur in a system of two ordinary differential equations. The time delay adds, in principle, an infinite number of other degrees of freedom, though in practice the number of active degrees of freedom involved in the observable laser dynamics depends on the size of $\tau_{R}$ compared to the intrinsic times scales in the laser operation.

We are not concerned with the dynamics of the optical phase in this system as it is removed by the use of the photodetector in the optoelectronic circuit. We write the amplitude as

$$
A(t)=\sqrt{I(t)} e^{i \phi(t)}
$$

and

$$
S(t)=\frac{2 \epsilon_{0} n^{\prime 2}}{\hbar \omega_{0}} I(t)
$$

is the photon density. Ignoring the spontaneous emission for now, we find

$$
\begin{aligned}
& \frac{d S(t)}{d t}=-\gamma_{c} S(t)+\Gamma g(N(t), S(t)) S(t) \\
& \frac{d N(t)}{d t}=\frac{J_{0}+J_{F}(t)}{e d}-\gamma_{s} N(t)-g(N(t), S(t)) S(t)
\end{aligned}
$$

when $g(N(t), S(t))$ is a phenomenological quantity. Over the range of variation of both $N(t)$ and $S(t)$ in our calculations, either a Taylor expansion about the fixed point at $d N(t) / d t=$ $d S(t) / d t=J_{F}=0$ or a simple ratio of terms incorporating the same information is adequate. If larger excursions in the dynamical variables were required, more details of the gain would be needed.

We expand $g(N(t), S(t))$ about the stationary CW operating condition, $\left(S_{0}, N_{0}\right)$ the dynamical fixed point of the laser in the absence of external feedback $J_{F}=0$. This gives us

$$
g(N, S)=g_{0}+g_{n}\left(N-N_{0}\right)+g_{p}\left(S-S_{0}\right) .
$$

Liu and Simpson [5] show how to experimentally estimate $g_{n}=$ $\partial g / \partial N>0$ and $g_{p}=\partial g / \partial S<0$. This operating point, defined by $d N(t) / d t=d S(t) / d t=J_{F}=0$, implies $\Gamma g_{0}=\gamma_{c}$ and fixes the bias current as $J_{0} / e d-\gamma_{s} N_{0}=g_{0} S_{0}$. By this normalization, our dynamical equations are independent of how far above threshold we set the laser except via indirect influence on the empirical differential gain parameters $g_{n}$ and $g_{p}$, which depend on the expansion point.

Transforming to dimensionless quantities $\mathrm{s}(t), \mathrm{n}(t)$, and $\mathcal{J}$ defined by $S(t)=S_{0}(1+\mathrm{s}(t)), N(t)=N_{0}(1+\mathrm{n}(t))$ and $\gamma_{s} N_{0}\left(\mathcal{J}+\mathcal{J}_{F}(t)\right)=\left(J_{0}+J_{F}(t)\right) / e d-\gamma_{s} N_{0}$, we have

$$
\begin{aligned}
& \frac{d \mathrm{~s}(t)}{d t}=-\gamma_{c}(1+\mathrm{s}(t))+\Gamma g(\mathrm{n}(t), \mathrm{s}(t))(1+s(t)) \\
& \frac{d \mathrm{n}(t)}{d t}=\gamma_{s}\left(\mathcal{J}+\mathcal{J}_{F}(t)\right)-\gamma_{s} \mathrm{n}(t)-g(\mathrm{n}(t), \mathrm{s}(t)) \frac{S_{0}}{N_{0}}(1+\mathrm{s}(t))
\end{aligned}
$$

and $S_{0} / N_{0}=\mathcal{J} \gamma_{s} / g_{0}$ by solving for the bias current at the $\mathrm{CW}$ operating point. The dimensionless gain $\tilde{g}=g / g_{0}$ is

$$
\tilde{g}(\mathrm{n}, \mathrm{s})=1+\frac{\gamma_{n}}{\mathcal{J} \gamma_{s}} \mathrm{n}-\frac{\gamma_{p}}{\gamma_{c}} \mathrm{~s}
$$


where $\gamma_{n}=g_{n} S_{0}$ and $\gamma_{p}=-\Gamma g_{p} S_{0}$. We have also investigated the gain in the form

$$
g(N, S)=\frac{g_{0}+g_{n}\left(N-N_{0}\right)}{1-g_{p}\left(S-S_{0}\right)}
$$

and found no change in our results over the dynamical range of our semiconductor lasers.

The natural relaxation oscillation frequency in this laser is $f_{R}=\sqrt{\gamma_{c} \gamma_{n}+\gamma_{s} \gamma_{p}} / 2 \pi$ with which we form the dimensionless time $\tau=t f_{R}$. Our dynamical equations for the optoelectronic feedback semiconductor laser now read

$$
\begin{aligned}
\frac{d \mathrm{~s}(\tau)}{d \tau}= & \frac{1}{f_{R}} \gamma_{c}(\tilde{g}(\mathrm{n}(\tau), \mathrm{s}(\tau))-1)(1+\mathrm{s}(\tau)) \\
\frac{d \mathrm{n}(\tau)}{d \tau}= & \frac{1}{f_{R}} \gamma_{s}\left(\mathcal{J}+\mathcal{J}_{F}(\tau)\right)-\gamma_{s} \mathrm{n}(\tau) \\
& -\gamma_{s} \tilde{g}(\mathrm{n}(\tau), \mathrm{s}(\tau)) \mathcal{J}(1+\mathrm{s}(\tau)) \\
\tilde{g}(\mathrm{n}, \mathrm{s})= & 1+\frac{\gamma_{n}}{\mathcal{J} \gamma_{s}} \mathrm{n}-\frac{\gamma_{p}}{\gamma_{c}} \mathrm{~s} .
\end{aligned}
$$

\section{B. Spontaneous Emission Noise}

Without laser noise, the optical phase entirely decouples from the dynamics. The situation is more complicated, however, once spontaneous emission is included. Equation (12) becomes

$$
\begin{array}{r}
\frac{d \mathrm{~s}(\tau)}{d \tau}=\frac{1}{f_{R}} \gamma_{c}(\tilde{g}(\mathrm{n}(\tau), \mathrm{s}(\tau))-1)(1+\mathrm{s}(\tau))+\frac{2}{\left|A_{0}\right| f_{R}^{1 / 2}} \\
\cdot(1+\mathrm{s})^{1 / 2}\left[F_{R} \cos \phi+F_{I} \sin \phi\right] .
\end{array}
$$

Even though both $F_{R}$ and $F_{I}$ are independent Gaussian processes, it is not entirely correct to replace the noise term on the right side with a single Gaussian noise process, because the optical phase $\phi$ is a dynamical variable stochastically perturbed by a noise term $F_{I} \cos \phi-F_{R} \sin \phi$, which is correlated with the intensity noise.

To derive the integration algorithm, we proceed as if we were integrating the original complex field with a complex noise term. The Langevin equation for $a=A /\left|A_{0}\right|$ reads

$$
\frac{d a}{d \tau}=F[a, \mathrm{n}]+\frac{1}{\left|A_{0}\right| f_{R}^{1 / 2}} F_{s p}
$$

with $F[\cdot]$ representing the deterministic dynamics. In the least difficult situation, a Gaussian white noise source with constant coefficients, the rule for the integration step is

$$
a(\tau+d \tau)=\tilde{a}(\tau+d \tau)+C^{1 / 2} \eta
$$

where $\tilde{a}$, the result of integrating the deterministic part $\tilde{a}=$ $\int F\left[a\left(t^{\prime}\right), \mathrm{n}\left(t^{\prime}\right)\right] d t^{\prime}$, the coefficient $C=d \tau R_{s p} / 2\left|A_{0}\right|^{2} f_{R}$, and $\eta$ is a complex-valued Gaussian random variable

$$
\eta=\eta_{1}+i \eta_{2} ; \quad \eta_{1,2} \in N(0,1) .
$$

We have for the intensity $\mathrm{s}+1=|a|^{2}$

$$
\mathrm{s}(\tau+d \tau)=\widetilde{\mathrm{s}}(\tau+d \tau)+C|\eta|^{2}+2 C^{1 / 2} \operatorname{Re}\left[\tilde{a}(\tau+d \tau) \eta^{*}\right] .
$$

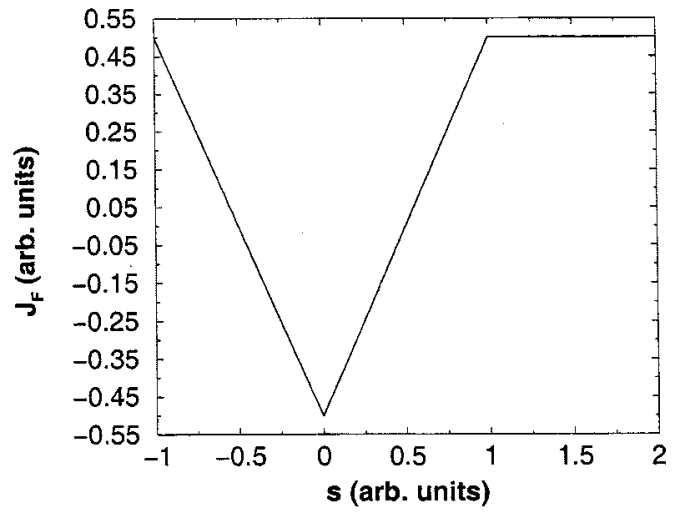

Fig. 2. The thresholded, inverted tent map (21), used as the nonlinear element of the electrooptical loop. $A=0.5, B=0.5$.

Separating the optical phase using $\tilde{a}=(\mathrm{s}+1)^{1 / 2} e^{i \phi}$

$\mathrm{s}(\tau+d \tau)=\tilde{\mathrm{s}}(\tau+d \tau)+C|\eta|^{2}+2 C^{1 / 2}(\tilde{\mathrm{s}}+1)^{1 / 2} \operatorname{Re}\left[e^{i \phi} \eta^{*}\right]$.

There is no preferred phase in the physics of the system. Because of this continuous symmetry, the phase angle is asymptotically uncorrelated with the intensity. In addition, the random variable $\eta$ is uniformly distributed in angle. Therefore, without loss of generality, we can define a new random variable $\xi=e^{-i \phi} \eta$, and this has exactly the same statistics as $\eta$, namely $\xi=\xi_{1}+$ $i \xi_{2}$, with each component being a random complex Gaussian as before. Thus, the explicit integration algorithm that we employ reads

$$
\begin{array}{r}
s(\tau+d \tau)=\tilde{\mathrm{s}}(\tau+d \tau)+2 C^{1 / 2}(\tilde{\mathrm{s}}(\tau+d \tau)+1)^{1 / 2} \\
\xi_{1}+C\left(\xi_{1}^{2}+\xi_{2}^{2}\right) .
\end{array}
$$

In the simulation of some toy model problems (not shown), this algorithm produced results for the distribution of intensity that were the same as integrating the complex equation of motion, whereas integrating the equivalent of (15), assuming a single noise source on the intensity, gave incorrect answers.

\section{Optoelectronic Feedback}

We investigated two functional forms for $\mathcal{J}_{F}(\tau)$. First, we considered straight linear feedback $\mathcal{J}_{F}(\tau)=\xi(\mathcal{J}+1)[\mathrm{s}(\tau-$ $\left.\left.\tau_{r}\right)+1\right], \tau_{r}=f_{R} \tau_{R}$, and second, the nonlinear inverted tent map function, as shown in Fig. 2. The functional form is

$$
\mathcal{J}_{F}(\tau)= \begin{cases}-\xi \mathrm{s}\left(\tau-\tau_{r}\right)-B, & \text { for } \mathrm{s}<0 \\ \xi \mathrm{s}\left(\tau-\tau_{r}\right)-B, & \text { for } 0 \leq \mathrm{s} \leq(\mathrm{A}+\mathrm{B}) / \xi \\ A, & \text { for } \mathrm{s} \geq(\mathrm{A}+\mathrm{B}) / \xi .\end{cases}
$$

This gives a nonlinear feedback current with three adjustable parameters A, B, and $\xi$. The positive parameter $\xi$ corresponds to the slope and $B$ to the value of the bottom peak of the inverted tent map. In both cases, we ensure that $\mathcal{J}_{0}+\mathcal{J}_{F}>0$ for the physical reason that current cannot be extracted from a laser.

A sample of the time series for each feedback choice is shown in Fig. 3. The additional complexity which arises with the inverted tent map nonlinearity is clear. 

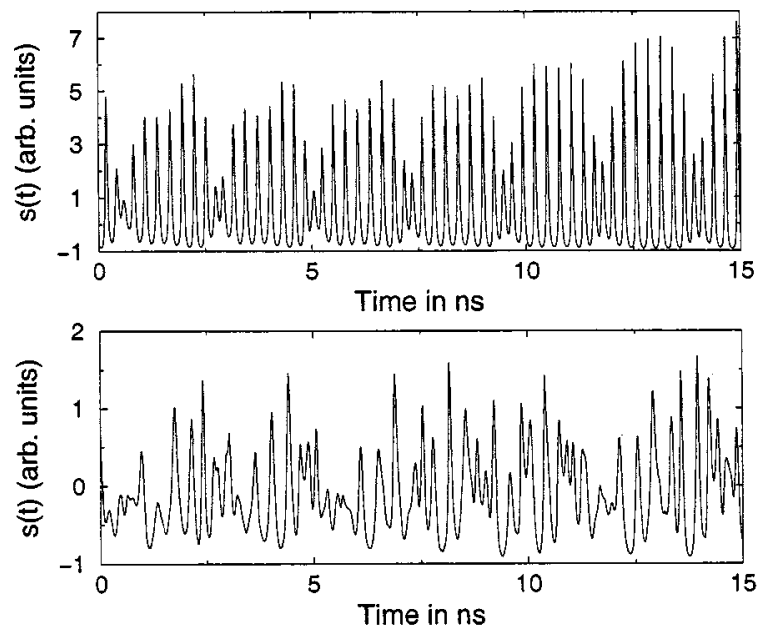

Fig. 3. Calculated time series from the chaotic optoelectronic feedback semiconductor laser: linear feedback (top), tent map feedback (bottom). These time series have $R_{s p}=0$.

For linear feedback with $\mathcal{J}_{F}(\tau)=\xi(\mathcal{J}+1)\left[\mathrm{s}\left(\tau-\tau_{r}\right)+1\right]$, there are significant periodic and quasi-periodic regimes interspersed with chaotic windows when the feedback time $\tau_{r}$ is close to a multiple of the natural oscillation time scale [6]. In Fig. 4, we show the bifurcation diagram for $\xi=0.15$ by plotting the extrema of the laser intensity $|A(t)|^{2}$ versus $\tau_{r}$. We exhibit the Lyapunov dimension evaluated from the time series of intensity for linear feedback in the same plot.

Lyapunov dimension is defined in terms of the ordered spectrum of Lyapunov exponents $\lambda_{1}>\lambda_{2}>\cdots$ by determining the integer $K$ at which the sum $\sum_{a=1}^{K} \lambda_{a}$ changes sign

$$
\sum_{a=1}^{K} \lambda_{a} \geq 0 ; \quad \sum_{a=1}^{K+1} \lambda_{a}<0
$$

and

$$
D_{L}=K+\frac{\sum_{a=1}^{K} \lambda_{a}}{\left|\lambda_{K+1}\right|} .
$$

The spectrum of Lyapunov exponents is determined using the same method as in [2]. This involves writing the effect of the numerical integration algorithm (in our case, a fourth-order predictor corrector) as a map operating on a very large state-space, consisting of the discretized ring of intensity states, representing the time-delayed feedback, and additional variables for the population inversion and time derivatives of intensity and population inversion. The very sparse Jacobian of this map may be computed analytically from the equations of motion, and implemented as a "Jacobian times vector" subroutine. The dynamics of the laser, along with the linearized dynamics of the tangent space, are simultaneously integrated, and the Lyapunov exponents stably extracted from the product of Jacobians with a standard recursive orthogonal/right-triangular (QR) matrix decomposition. We estimate the Lyapunov dimension from the calculated spectrum of Lyapunov exponents by first setting all exponents with absolute values smaller than a certain cutoff to zero and then applying (23). This is necessary, because the convergence of the calculated Lyapunov exponents to their limit values a)

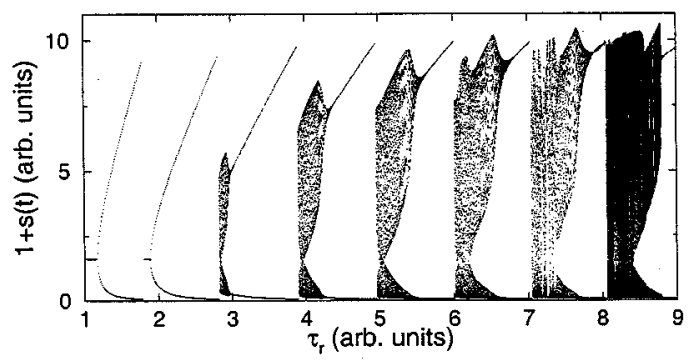

b)

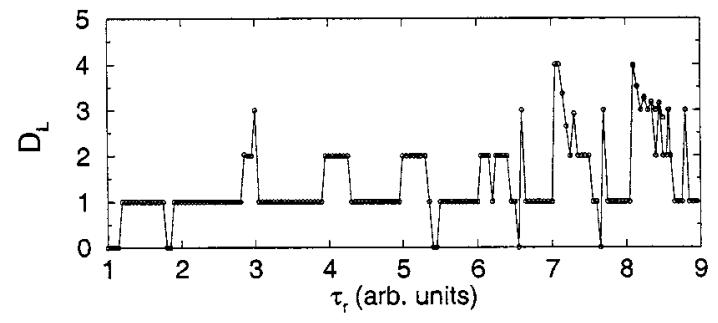

Fig. 4. (a) Bifurcation diagram and (b) estimated Lyapunov dimension $D_{L}$ for the semiconductor laser with linear feedback. This plot uses numerical simulations with $R_{s p}=0$.

is slow for high-dimensional systems like this one. For nonchaotic attractors the largest exponent is zero and the dimension is determined by the number of zero Lyapunov exponents. In this case small deviations of the calculated Lyapunov exponents from their true zero value can change the estimated dimension dramatically.

The frequent truncations of the bifurcation sequence, exhibited in Fig. 4, as well as the existence of multistable regimes, which we found in our numerical simulations, indicate the complexity of the detailed bifurcation diagram for the semiconductor laser rate equations with optoelectronic feedback. In Fig. 4, we see that the typical stages in the bifurcation sequence for the linear feedback are a Hopf bifurcation of the CW steady state with a Lyapunov dimension of zero to a limit cycle with $D_{L}=1$ and, subsequently, a second bifurcation to a two-torus, corresponding to quasi-periodic motions with $D_{L}=2$. Upon increasing the control parameter $\tau_{r}$ further, we observe chaotic motion. Varying the feedback strength $\xi$ while keeping the time delay $\tau_{r}$ fixed one obtains the same stages in the bifurcation sequence. This indicates a quasi-periodic route to chaos, which agrees well with results from analytic bifurcation analyses [7], [8].

In Fig. 5, we present a more detailed look at a typical bifurcation sequence for the linear feedback by showing the power spectral density in the first column and the Poincare section in the second column for time delays of $\tau_{r}=9.0,8.6,8.58$, and 8.1 (A-D), respectively. The frequencies are given in units of the relaxation oscillation frequency of the laser without feedback. We use time-delay embedding coordinates and obtain the Poincaré section by defining a 3-D hyperplane, e.g., with coordinates $(x, y, z)$ for definiteness, in a 4-D embedding space. The graphs in the second column present the projection of the Poincaré section along the $z$ axis. By means of Hopf bifurcations, limit cycle attractors are created, and in the first column Fig. 5(A) shows the power spectral density, and in the second column the Poincaré section of such an attractor. Note that the frequency of the limit cycle oscillations is on the order of the 

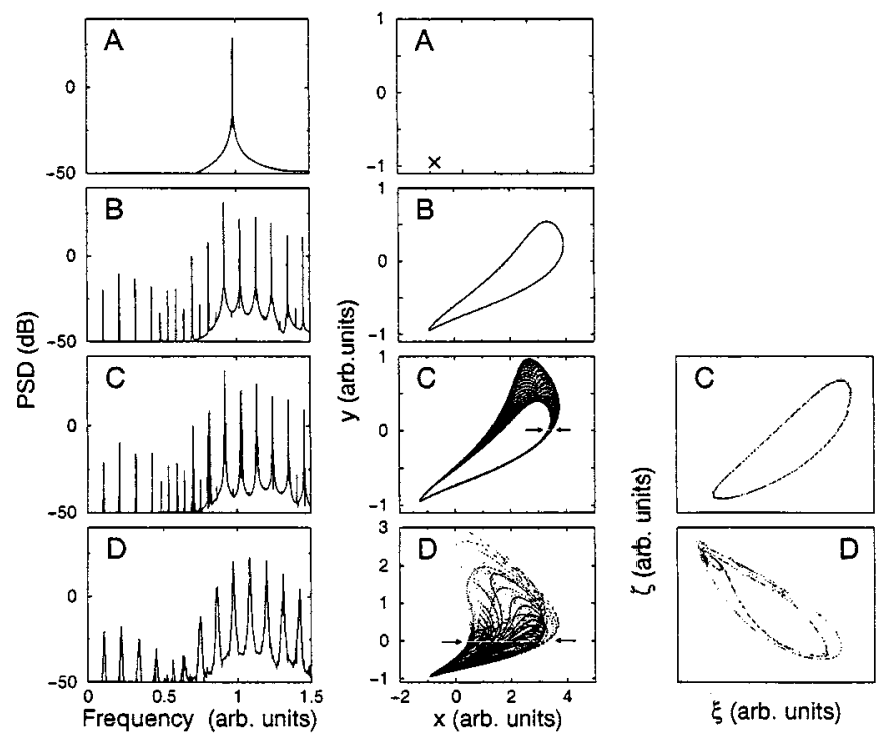

Fig. 5. Numerical evidence for the quasi-periodic route to chaos for the linear feedback. Power spectral density (first column) and projection of the Poincaré section onto the $x-y$-plane (second column) for the case of: (A) limit cycle oscillations; (B) quasi-periodic oscillations on a two-torus; (C) oscillations on a three-torus; and (D) chaos. For the three-torus (C) and chaos (D), we display in the third column a very thin slice through the 3-D Poincare section at the location indicated by the arrows (second column).

natural relaxation oscillation frequency. A decrease of the delay time leads to a destabilization of the limit cycle and a two-torus is created Fig. 5(B). A second incommensurate frequency appears with a value on the order of the inverse of the delay time in the feedback, significantly slower than the relaxation oscillation frequency. The toroidal nature of the attractor can clearly be seen in the Poincaré section. At a slightly shorter time delay, a three-torus is found Fig. 5(C). A close examination of the spectrum reveals the appearance of a third incommensurate frequency, with a value significantly lower than both the relaxation oscillation frequency and the frequency corresponding to a round-trip time. To discern the three-torus nature of the attractor, we display in the third column a very thin slice through the 3-D Poincare section. The location of the slice is indicated by the arrows in the second column of Fig. 5(C). It shows, that the closed curve of Fig. 5(B), which represents a two-torus attractor, bifurcates to a two-torus in the section, corresponding to a three-torus attractor. Finally, when entering the chaotic regime as in Fig. 5(D), we observe a breakup of the three-torus, as well as the development of a broadband background in the spectrum.

The estimated Lyapunov dimensions for (A)-(D), using a cutoff of $1 \cdot 10^{-4}$, are $D_{L}=1.00, D_{L}=2.00, D_{L}=3.00$, and $D_{L}=3.95$ respectively. For case (D), the largest Lyapunov exponent is clearly positive, whereas for the other three cases, the absolute value of the largest Lyapunov exponent is smaller than the cutoff. This agrees well with the quasi-periodic route to chaos and shows that Fig. 5(D) does, indeed, correspond to chaotic dynamics of the feedback laser.

For the purpose of communication with chaos, it suffices to find the parameter regimes corresponding to chaotic oscillations. In Fig. 4, we see that with linear feedback $D_{L}$ rises above three only for $\tau_{r}>7$. In contrast, the inverted tent map feedback a)

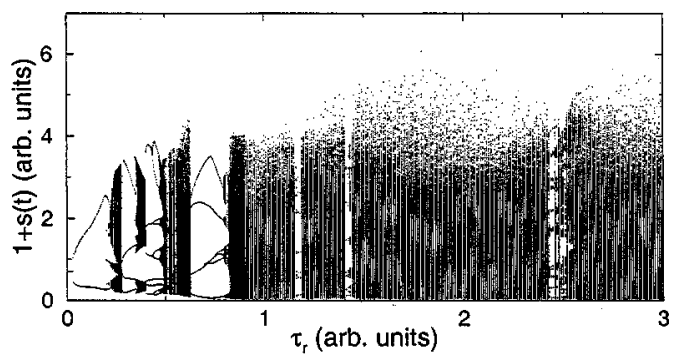

b)

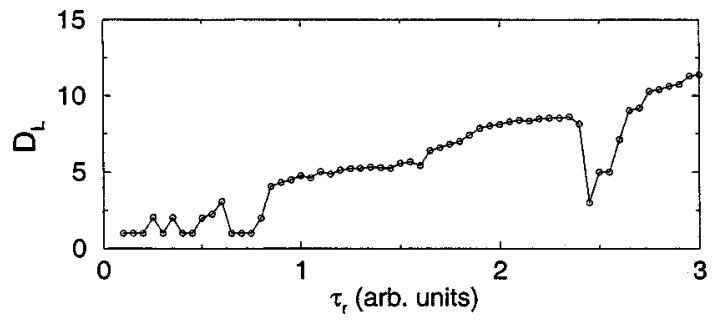

Fig. 6. Bifurcation diagram and estimated Lyapunov dimensions for the semiconductor laser with: (a) inverted tent map feedback and (b) no spontaneous emission noise.

function gives high-dimensional chaos over most time-delays. $D_{L}$ is large, and usually noninteger for $\tau_{r}>0.5$, as shown in Fig. 6. Linear feedback does not require additional electronics in the optoelectronics loop, so it is easier to implement experimentally.

Although with time-delayed feedback the dimension of this dynamical system can be quite high, it is still substantially less than that for a semiconductor laser subjected to optical feedback in many regimes as represented by the Lang-Kobayashi equations. Furthermore, the autocorrelation time scale of the dynamics of the optical-phase-dependent Lang-Kobayashi system is at least an order of magnitude faster than in our system. Optical feedback is governed by the large photon decay rate $\gamma_{c} \approx$ $3 \times 10^{11} s^{-1}$ instead of the spontaneous carrier relaxation rate $\gamma_{s} \approx 1.5 \times 10^{9} s^{-1} \cdot \gamma_{c}$ is too fast for simple experimental techniques to give time-resolved measurements, while the time scale of trajectories in our optoelectronic feedback allow for time trace measurements using fast oscilloscopes.

\section{SYNCHRONIZATION}

\section{A. Coupling the Transmitter and Receiver}

As a prelude to using two of our laser setups for communication we investigate the synchronization of their oscillations. In regimes where the lasers synchronize, the action of the receiver laser reflects in a deterministic way the oscillations of the transmitter, and thus, even though they each can be chaotic, one can recognize the effect of modulation at the transmitter and demodulate this at the receiver.

To investigate synchronization, we couple two lasers which we denote as the transmitter with dynamical variables $\mathrm{n}_{T}(t), \mathrm{s}_{T}(t)$ and the receiver with $\mathrm{n}_{R}(t), \mathrm{s}_{R}(t)$, calling time $t$ again. We connect the transmitter to the receiver unidirectionally by transmitting $J_{T}(t)=\left|A_{T}(t)\right|^{2}$ to the receiver. At the receiver we multiply $J_{T}(t)$ by $0 \leq c \leq 1$ and add $c J_{T}(t)$ to the receiver intensity $J_{R}(t)$ multiplied by $1-c$. The current signal 
TABLE I

Dynamical Variables, Definitions, AND Numerical Values of Parameters

\begin{tabular}{|c|c|c|}
\hline Symbol & Value & Description \\
\hline $\begin{array}{c}\mathrm{s}(\tau) \\
\mathrm{n}(\tau) \\
\tilde{g}(\mathrm{n}(\tau), \mathrm{s}(\tau)) \\
\mathcal{J}_{\mathcal{J}}(\tau)\end{array}$ & & $\begin{array}{l}\text { dimensionless intracavity photon density } \\
\text { dimensionless carrier density } \\
\text { dimensionless gain coefficient } \\
\text { dimensionless feedback current }\end{array}$ \\
\hline $\begin{array}{c}\mathcal{J} \\
\gamma_{s} \\
\gamma_{n} / \mathcal{J} \\
\gamma_{p} / \mathcal{J} \\
\gamma_{c} \\
\xi \\
f_{R}=\left(\gamma_{c} \gamma_{n}+\gamma_{s} \gamma_{p}\right)^{1 / 2} / 2 \pi \\
R_{s p} /\left|A_{0}\right|^{2}\end{array}$ & $\begin{array}{c}2 / 3 \\
1.458 \times 10^{9} s^{-1} \\
2.0 \times 10^{9} s^{-1} \\
3.6 \times 10^{9} s^{-1} \\
3.6 \times 10^{11} s^{-1} \\
0.15 \\
3.5 \times 10^{9} s^{-1} \\
9.55 \times 10^{6} s^{-1}\end{array}$ & $\begin{array}{l}\text { bias current at fixed point } \\
\text { spontaneous carrier decay rate } \\
\text { gain variation with carrier density } \\
\text { gain variation with photon density } \\
\text { photon decay rate } \\
\text { feedback coefficient } \\
\text { relaxation oscillation frequency } \\
\text { spontaneous emission noise variance }\end{array}$ \\
\hline
\end{tabular}

into the receiver is, thus, $c J_{T}(t)+(1-c) J_{R}(t)$, leading to the coupled equations of motion

$$
\begin{aligned}
f_{R} \frac{d \mathrm{~s}_{T}(t)}{d t}= & \gamma_{c}\left(\tilde{g}\left(\mathrm{n}_{T}(t), \mathrm{s}_{T}(t)\right)-1\right)\left(1+\mathrm{s}_{T}(t)\right) \\
& +2 \sqrt{\frac{2 \epsilon_{0} n^{\prime 2}\left(1+\mathrm{s}_{T}(t)\right)}{S_{0} \hbar \omega_{0}}} F_{S} \\
f_{R} \frac{d \mathrm{n}_{T}(t)}{d t}= & \gamma_{s}\left(\mathcal{J}+\mathcal{J}_{F T}(t)\right)-\gamma_{s} \mathrm{n}_{T}(t) \\
& -\gamma_{s} \tilde{g}\left(\mathrm{n}_{T}(t), \mathrm{s}_{T}(t)\right) \mathcal{J}\left(1+\mathrm{s}_{T}(t)\right)
\end{aligned}
$$

and

$$
\begin{aligned}
f_{R} \frac{d \mathrm{~s}_{R}(t)}{d t}= & \gamma_{c}\left(\tilde{g}\left(\mathrm{n}_{R}(t), \mathrm{s}_{R}(t)\right)-1\right)\left(1+\mathrm{s}_{R}(t)\right) \\
& +2 \sqrt{\frac{2 \epsilon_{0} n^{\prime 2}\left(1+\mathrm{s}_{R}(t)\right)}{S_{0} \hbar \omega_{0}}} F_{S} \\
f_{R} \frac{d \mathrm{n}_{R}(t)}{d t}= & \gamma_{s}\left(\mathcal{J}+\left[c \mathcal{J}_{F T}(t)+(1-c) \mathcal{J}_{F R}(t)\right]\right)-\gamma_{s} \mathrm{n}_{R}(t) \\
& -\gamma_{s} \tilde{g}\left(\mathrm{n}_{R}(t), \mathrm{s}_{R}(t)\right) \mathcal{J}\left(1+\mathrm{s}_{R}(t)\right) .
\end{aligned}
$$

In the case of linear feedback, for example

$$
\begin{aligned}
& \mathcal{J}_{F T}=\xi(\mathcal{J}+1)\left[\mathrm{s}_{T}\left(t-\tau_{r}\right)+1\right] \\
& \mathcal{J}_{F R}=\xi(\mathcal{J}+1)\left[\mathrm{s}_{R}\left(t-\tau_{r}\right)+1\right] .
\end{aligned}
$$

The net current injected into the receiver semiconductor laser element is

$$
\xi(\mathcal{J}+1)\left[\operatorname{cs}_{T}\left(t-\tau_{r}\right)+(1-c) \mathrm{s}_{R}\left(t-\tau_{r}\right)+1\right] .
$$

We see that for all $c$, the identity solution $\mathrm{s}_{T}(t)=\mathrm{s}_{R}(t)$ and $\mathrm{n}_{T}(t)=\mathrm{n}_{R}(t)$ is always possible. It is not necessarily a stable solution, and only when it is stable do we say that the transmitter and receiver are synchronized. When $c=1$, the receiver is being run open loop, as its optoelectronic feedback is disconnected.

The quality of the synchronization when $R_{s p} \neq 0$ is shown in Fig. 7, where we plot $\mathrm{s}_{T}(t)$ versus $\mathrm{s}_{R}(t)$ coming from calculations where $c=1$ and $R_{s p}$ is set to the standard value listed in

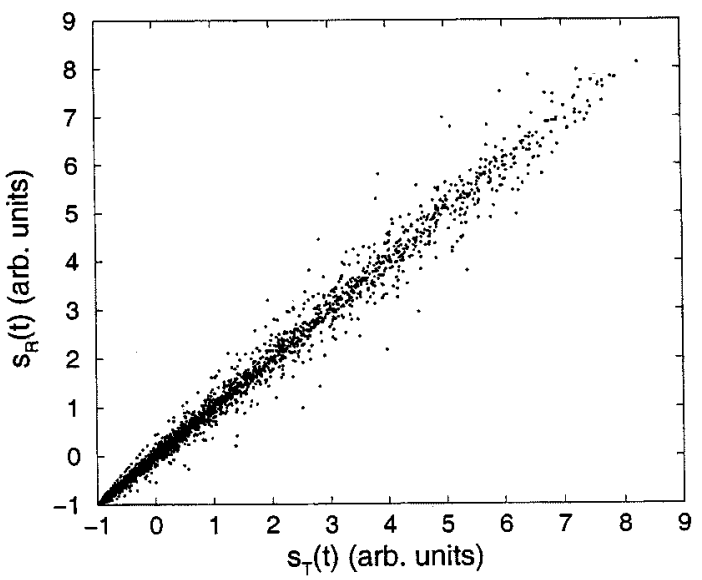

Fig. 7. Synchronization of two optoelectronic feedback semiconductor lasers, where $R_{s p} \neq 0$.

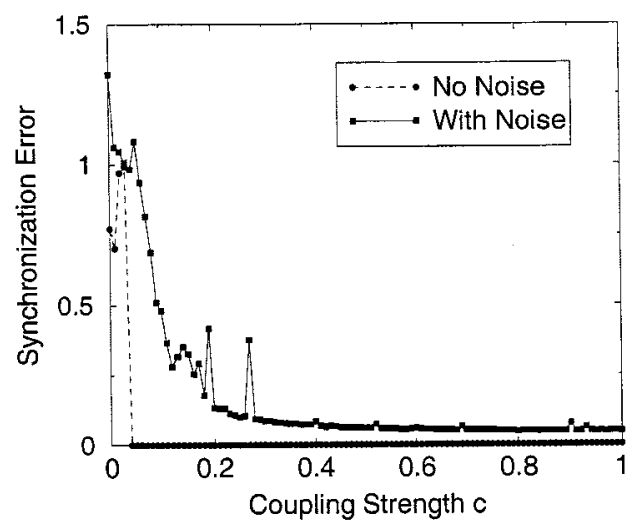

Fig. 8. Synchronization error in coupling of two optoelectronic feedback semiconductor lasers as a function of the coupling coefficient $c . R_{s p}=0$ (circles), $R_{s p} \neq 0$ (squares).

Table I. As a function of $c$, we show in Fig. 8 the synchronization error

$$
\mathcal{E}=\frac{\left\langle\left|\mathrm{S}_{T}(t)-\mathrm{s}_{R}(t)\right|\right\rangle}{\left\langle\mathrm{S}_{T}(t)\right\rangle}
$$


where $\langle\cdot\rangle$ means time average

$$
\langle f(t)\rangle \equiv \frac{1}{T} \int_{0}^{T} d t f(t)
$$

and the integration is over the total observed or calculated time series.

With $R_{s p}=0$, we see in Fig. 8 that the semiconductor lasers synchronize essentially perfectly for $c \geq 0.1$ and undergo a rapid transition to the unsynchronized state for $c \leq 0.1$. The desynchronization for $R_{s p} \neq 0$ in Fig. 8 is partly a result of spontaneous emission, but also a result of the intrinsic dynamics of the coupled systems.

\section{B. Parameter Mismatch Between Transmitter and Receiver}

The transmitter and receiver systems are inevitably different from each other. The many parameters characterizing the semiconductor lasers or the optoelectronic feedback loops can never be identical in a real system. In this situation, identity synchronization $\mathrm{s}_{T}(t)=\mathrm{s}_{R}(t)$ and $\mathrm{n}_{T}(t)=\mathrm{n}_{R}(t)$ is not a mathematical solution of our model equations. Nonetheless, the deviation from the identity solution can be small over some range of parameters in analog devices such as the ones we envision here. Over some range of parameters, one might expect to achieve generalized synchronization [9], in which the transmitter and receiver dynamical variables are definite, typically unknown, nonlinear functions of each other. There is a set of experiments on a quite different class of laser systems [10], [11], which suggest that the transition from the totally unsynchronized state of two coupled nonlinear system through generalized synchronization to identity synchronization is smooth.

We have evaluated the effect of various parameter mismatches between the transmitter and the receiver on the quality of synchronization, and have computed $\mathcal{E}$ as a function of

$$
\begin{aligned}
& \left(\gamma_{\mathrm{c}}^{R}-\gamma_{\mathrm{c}}^{T}\right) / \gamma_{\mathrm{c}}^{T} \\
& \left(\gamma_{\mathrm{s}}{ }^{R}-\gamma_{\mathrm{s}}^{T}\right) / \gamma_{\mathrm{s}}{ }^{T} \\
& \left(\gamma_{\mathrm{n}}{ }^{R}-\gamma_{\mathrm{n}}^{T}\right) / \gamma_{\mathrm{n}}{ }^{T} \\
& \left(\gamma_{\mathrm{p}}{ }^{R}-\gamma_{\mathrm{p}}^{T}\right) / \gamma_{\mathrm{p}}{ }^{T}
\end{aligned}
$$

In Fig. 9, we display the value of $\mathcal{E}$ for mismatches in $\gamma_{c}, \gamma_{s}, \gamma_{p}$ and $\gamma_{n}$ for $c=0.4$ and for $c=1$. In these calculations $R_{s p} \neq 0$, so even at perfect parameter matching, there is a nonzero $\mathcal{E}$. While the error is tolerable for a $5 \%$ or so mismatch at $c=1$, for $c=0.4$ the error is large for almost all mismatches.

To investigate the synchronization error associated with differences in the properties of the two feedback loops, we investigated $\mathcal{E}$ as a function of

$$
\left(\xi^{R}-\xi^{T}\right) / \xi^{T}
$$

and

$$
\left(\tau_{r}^{R}-\tau_{r}^{T}\right) / \tau_{r}^{T}
$$

Fig. 10 displays $\mathcal{E}$ versus the mismatch in the feedback rate $\xi$. Here, we display $\mathcal{E}$ for $c=0.4,0.7$ and 0.95 . It is clear that, as $c$ increases, the error due to mismatches becomes small. This

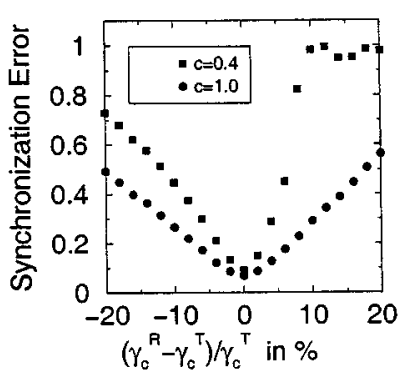

(a)

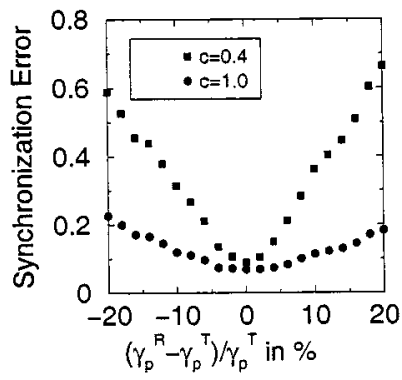

(c)

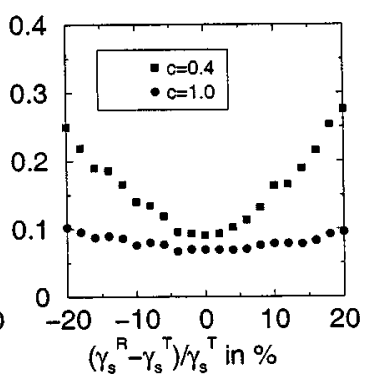

(b)

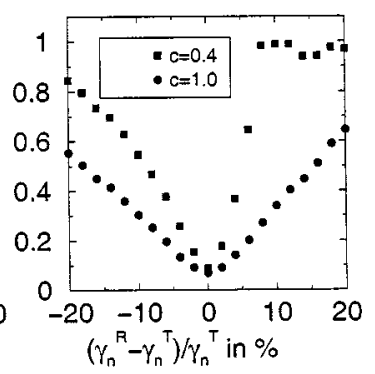

(d)
Fig. 9. Synchronization error from mismatches in $\gamma_{c}$ (upper left), $\gamma_{s}$ (upper right), $\gamma_{p}$ (lower left), and $\gamma_{n}$ (lower right).

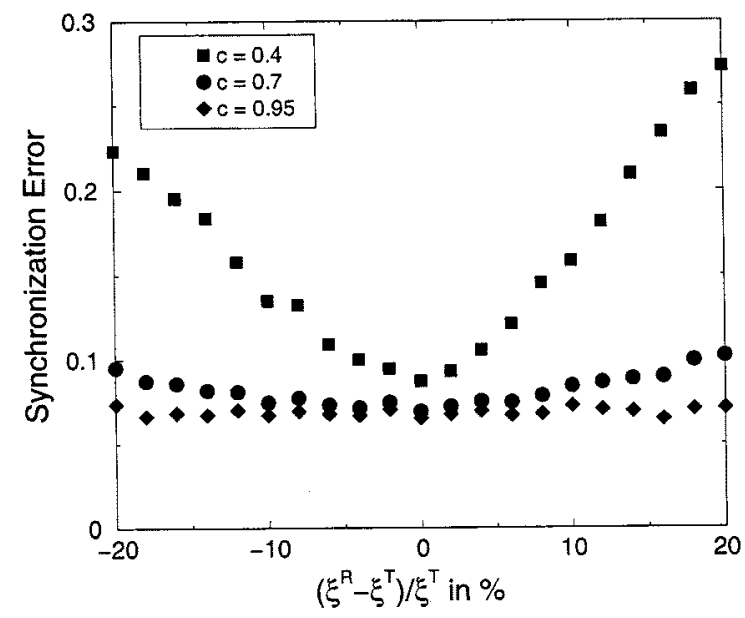

Fig. 10. Synchronization error from mismatches in $\xi$.

again points to the use of a $c=1$ open-loop receiver configuration. Fig. 11 displays the synchronization error $\mathcal{E}$ as a function of the mismatch in feedback delay $\tau_{r}$.

From this, we see that feedback rate mismatch does not significantly affect the synchronization quality while the influence of the delay-time mismatch is very significant. Also, from Fig. 11, we see that the synchronization error oscillates with the increase of the delay-time mismatch. The oscillation period corresponds to the time interval between two pulses in the output of a chaotic pulse train. So, each time the mismatch in the delay time increases to the amount of a pulse-to-pulse interval, the second pulse will substitute the first pulse and the synchronization error will be reduced. Because of the significant effect of the delay-time mismatch on the synchronization, we need to adjust the delay time in the transmitter and receiver feedback loops very well. 


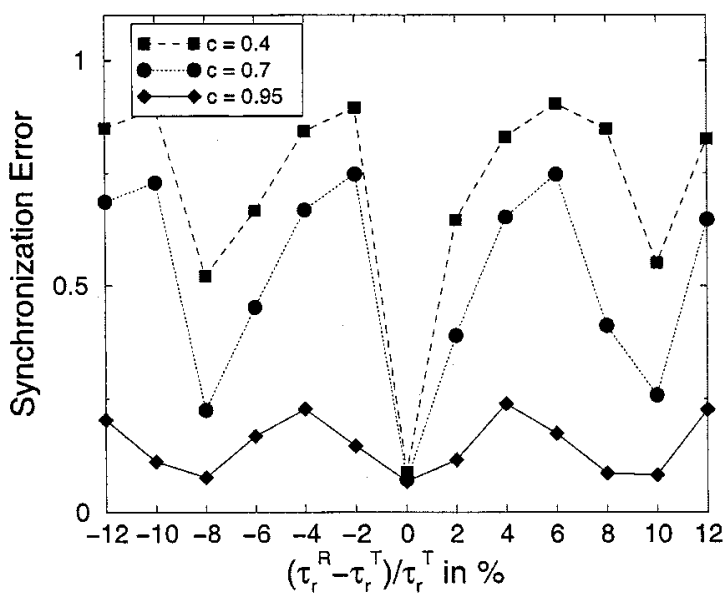

Fig. 11. Synchronization error from mismatch in feedback time delay $\tau_{r}$.

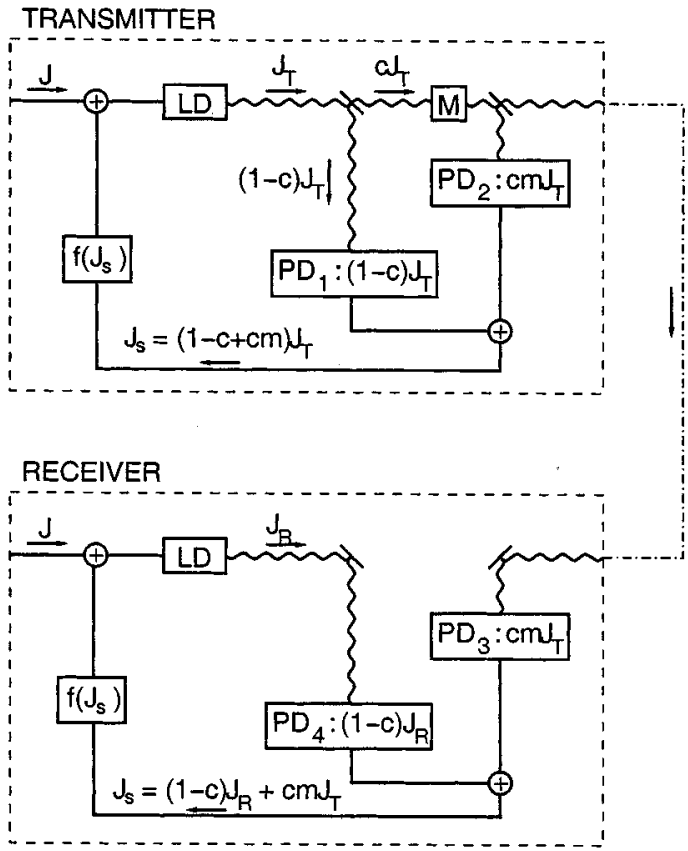

Fig. 12. Schematic of transmitter and receiver systems which works for any value of the modulation. $P D_{1} \cdots P D_{4}$ are photodiodes, providing electrical signal proportional to input intensity. (Symbol $P D_{k}: J$ is shorthand for current $J$ coming from photodiode number $k$.) We use $M$ to denote an electrooptical modulator which multiplicatively modulates information onto the intensity.

From these evaluations of $\mathcal{E}$ as a function of parameter mismatch, we see that the open-loop $c=1$ configuration is preferred. It is more robust to parameter mismatch, and it has excellent synchronization in the perfectly mismatched state. Since the coupled systems are quite sensitive to time-delay mismatches when $c \neq 1$, we prefer this open-loop configuration.

\section{COMMUNICATIONS}

To assure that we can maintain synchronization of the transmitter and receiver while using an arbitrary modulation amplitude for arbitrary $0 \leq c \leq 1$, we have adopted the scheme shown in Fig. 12: identical synchronization is a solution of the dynamical equations with and without modulation. We use a multiplicative modulation scheme which can be realized by $\mathrm{LiNiO}_{3}$ modulators at frequencies well above a gigahertz. This modulator attenuates the intensity roughly proportional to the voltage imposed across it, so we can represent the intensity leaving the modulator as $m(t) J_{T}(t) . m(t)$ is the message; it can be analog, digital, voice, or data. We transport the field down the communications channel, resulting in a received intensity $J_{C}(t)=$ $\mathrm{cm}(t) J_{T}(t)$. Recall, we are concerned with intensities alone as the optical phases at transmitter and receiver need not be matched in our scheme.

The receiver detects an intensity $J_{C}(t)=c m(t) J_{T}(t)$ and adds to that $(1-c) J_{R}(t)$ in its nonlinear feedback loop. Identity synchronization $J_{T}(t)=J_{R}(t)$ is still a solution to the coupled equations. At the receiver, one estimates the message by

$$
m_{E}(t)=\frac{J_{C}(t)}{c J_{R}(t)}
$$

or in the notation depicted in Fig. 12

$$
m_{E}(t)=\frac{(1-c)}{c} \frac{P D_{3}}{P D_{4}}
$$

The symbol $P D_{k}$ stands for the intensity coming from photodetector number $k$.

It is not necessary to have two physical photodiodes in the transmitter laser, as one may calibrate the photodiode gain, as well as the modulator gain and offset to produce the same function. We show the diagram Fig. 12 to emphasize the required symmetry between transmitter and receiver. The receiver requires two distinct photodiodes. Notice that nowhere in the system does the optical phase or electric field polarization explicitly enter the dynamics. Photodiodes are intensity-only devices, and the intensity modulator has a simple multiplicative influence on the intensity as well. Thus, this scheme ought to be insensitive to the difficult problems of matching optical path lengths of transmitter and receiver, as well as being insensitive to environmental fluctuations in the optical phase and polarization state as the signal propagates through the communications channel, e.g., a fiber optic cable. An atmospheric channel, however, could have an effect on the intensity and, thus, the communication performance.

\section{A. Communications: No Channel Noise}

To achieve communication using our multiplicative modulation strategy, we transmit digital nonreturn-to-zero bits so that a "one" is represented by $m=e^{+B}$ and a "zero" by $m=e^{-B}$. This is transmitted in a fixed bit time $T_{b}$. The message is then imposed on the received intensity $m(t) J_{T}(t)$, which is always positive and bounded away from zero in our construction.

At the receiver, we form the quantity

$$
\begin{aligned}
\log m_{E}(t) & =\log \left(J_{C}(t) / J_{R}(t)\right) \\
& =\log m(t)+\log \left(J_{T}(t) / J_{R}(t)\right)
\end{aligned}
$$

to demodulate this information. This expression for the estimated message signal $m_{E}(t)$ emphasizes the error which is introduced by a failure to have a synchronized transmitter and receiver. Fig. 13 shows the demodulation error as a function of $c$. This becomes just a normalized synchronization error when we 

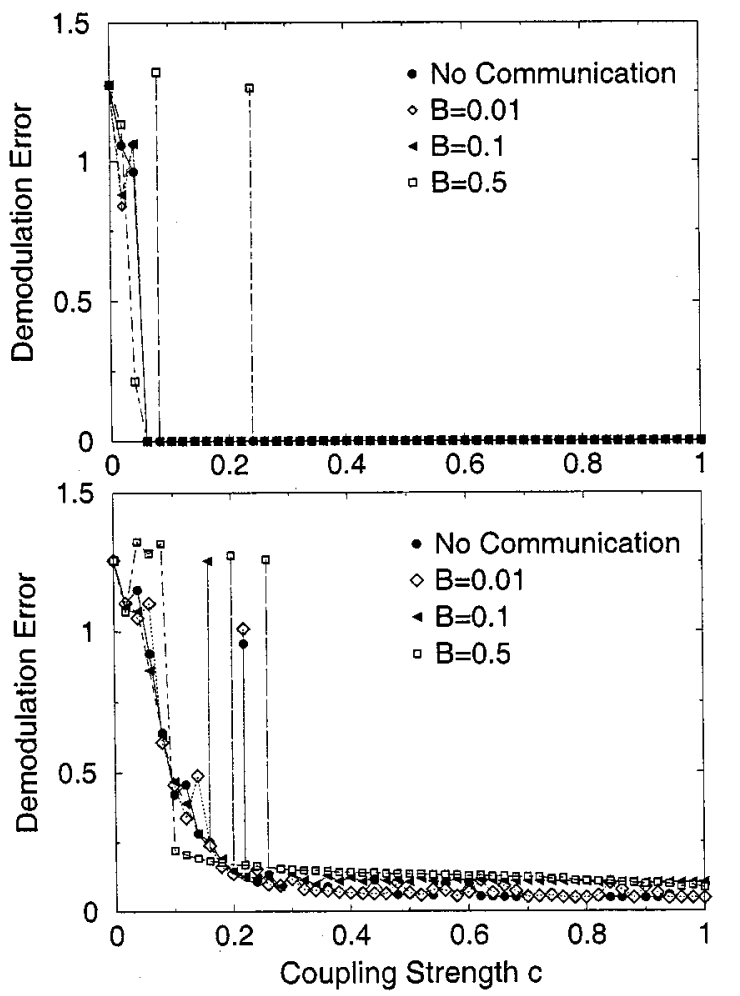

Fig. 13. Average demodulation error $\left\langle\log \left(J_{R} / J_{T}\right)\right\rangle$ with varying bit size $B$, $T_{b}=1$ and no channel noise as a function of the coupling strength between transmitter and receiver. $m=e^{ \pm B}$ for a " 1 " or a " 0 " respectively. $B=0$ or $m=1$ means no message is modulated onto the transmitter signal. We display both: (a) the case $R_{s p}=0$ and taking spontaneous emission noise into account (b) $R_{s p} \neq 0$. This figure demonstrates that with the communication scheme of Fig. 12 large modulation and small modulation produce small errors.

have a transmission with no modulation $-B=0$ or $m=1$, and is displayed in Fig. 8 above. Fig. 13 shows

$$
\frac{\left\langle\left(\log m_{E}(t)-\log m(t)\right)^{2}\right\rangle}{\left\langle\left(\log J_{T}(t)\right)^{2}\right\rangle}=\frac{\left\langle\left(\log J_{R}(t)-\log J_{T}(t)\right)^{2}\right\rangle}{\left\langle\left(\log J_{T}(t)\right)^{2}\right\rangle}
$$

as a function of the coupling constant $c$. Without spontaneous emission noise and above a critical coupling, essentially perfect synchronization is achieved [Fig. 13(a)]. Most strikingly, the performance is independent of the size and the period of the modulation, with neither the error nor critical coupling changing substantially. In other words, the system is stable to large amplitude modulation. As in [2], we have found this result to hold over a range of bit rates an order of magnitude below and above the result presented here, as well as for nonrepeating analog messages. We mention that successful decoding can take place when the modulation is not visible to conventional techniques, if, for instance, chaotic signal-masking is desirable. Fig. 14 shows a sample of transmitted optical intensity with $B=0.1$ size modulation, which is significantly smaller than the natural dynamic range of the oscillations. The modulation signal is not clearly visible in the time series or power spectrum compared to an unmodulated signal, yet the recovery of the modulated signal is perfect in the absence of noise. From a dynamical point of view, the fact that the system is stable even to large modulation depth (though this is not necessary for signal recovery) is more striking. The role of including spontaneous emission fluc-
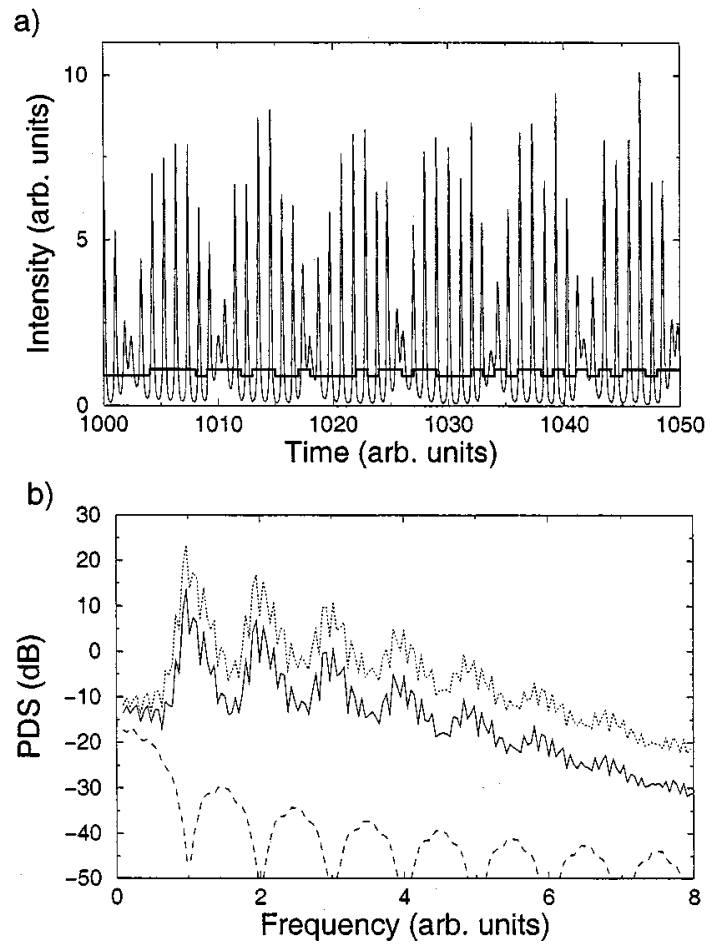

Fig. 14. (a) Sample of transmitted modulated intensity, superimposed with modulating signal. (b) Power spectral densities of transmitter laser intensity signal pre-modulation (dashed), post-modulation (solid), and modulation signal (dashed). The dotted trace has been shifted by $+10 \mathrm{~dB}$ for greater visibility, it would otherwise nearly superpose the solid trace.

tuations can be seen in Fig. 13(b), raising the error floor as expected.

\section{B. Communications: Channel Noise}

We now turn to the performance in a noisy channel. While not anticipating significant noise for transmission through an optical fiber, the situation may not be quite the opposite for transmission through the atmosphere. To represent the noise characteristics of the channel, we use a multiplicative Gaussian stochastic model for channel noise, which is considered a good first approximation [12] to the characteristics of a noisy optical channel. The physical picture behind this characterization is that the signal which enters the receiver laser, after traversing the channel, is $c J_{C} e^{\eta(t)}$, with $\eta(t)$ being a Gaussian white process.

Chen and Yao have shown [13], [14] that it is critical to use proper stochastic integration algorithms to simulate chaotic communications schemes. Unfortunately, it seems there are no satisfactory algorithms for integrating stochastic differential equations with substantial nonlinearities associated with the stochastic variable in the vector field. We proceed by adding an additional dynamical element by letting $\eta(t)$ be a new independent variable with Brownian dynamics. This is expressed via the Langevin equation for $\eta(t)$

$$
\frac{d \eta(t)}{d t}=-\frac{1}{\tau_{c}} \eta+\mu \frac{1}{\sqrt{\tau_{c}}} N(0,1)
$$

where $N(0,1)$ is a Gaussian white process with zero mean and variance unity. This produces, in our noise variable $\eta(t)$, a Gaussian process with correlation time $\tau_{c}$ and variance $\mu^{2}$. The 
value of $\tau_{c}$ depends on the physics underlying the scattering in the channel. For clear air it is much less than a nanosecond, the characteristic time of dynamics in our laser. On time scales relevant to the operation of our lasers, $\eta$ is nearly white in this setting. With strong particle scattering, it may not be so. We originally introduced this device in order to put the set of equations in a suitable form (linear in the stochastic variable) for the numerical algorithm we employ [15]. Now we can use it to more faithfully represent the actual physics of the channel.

The receiver measures $J_{C} e^{\eta(t)}$ and the demodulator reconstructs the estimated message $m_{E}(t)$ as

$$
\begin{aligned}
\log m_{E}(t) & =\log \left(J_{C}(t) / J_{R}(t)\right) \\
& =\log m(t)+\log \left(J_{T}(t) / J_{R}(t)\right)+\eta(t) .
\end{aligned}
$$

We average $\left\langle\log m_{E}(t)\right\rangle_{T_{b}}$ over a bit time $T_{b}$, and sample the signals every $T_{s}$. If this average is greater than zero, then we call it a "one." If it is less than zero, we call it a "zero." In our studies, $T_{b} / T_{s}=10$. In these variables, the message $\log m$ is contaminated with additive stochastic noise through $\eta$, as well as by any deviation in synchronization $\log \left(J_{T} / J_{R}\right)$.

As is conventional in communication theory, we report the bit error rate (BER) versus the "energy per bit" divided by the spectral density of the noise $E_{b} / \mathcal{N}_{0}$. In our circumstances, it is not clear what the proper energy per bit $E_{b}$ means, but if one ignores the chaotic carrier for the moment and looks only at $\log m$ as the signal contaminated by white Gaussian additive noise, then $E_{b}=B^{2} T_{b}$ and the noise density $\mathcal{N}_{0}=\left\langle\eta^{2}\right\rangle T_{s}$. Fig. 15 summarizes our results for BER versus $E_{b} / \mathcal{N}_{0}$ calculated with $5 \times 10^{5}$ bits, with bit time $T_{b}=1$ in dimensionless units, and bit size $B=0.1$, over a range of noise strengths. This bit size is the same as the one shown in Fig. 14 . Generally, $E_{b} / N_{0}$ parameterizes the performance reasonably well, as it does for conventional linear communications. We include reference curves corresponding to the perfect direct detector, i.e., estimating BER, assuming no synchronization error $\log \left(J_{T} / J_{R}\right)=0$, in (34). Above a certain $c \approx 0.5$, the synchronization threshold, communication performance is good with vanishing error rate as the noise strength declines. The curves for chaotic synchronization lie slightly to the right of the "direct signaling" curve because some channel noise enters the feedback loop of the receiver. In many regimes, however, this does not cause global desynchronization and, thus, complete loss of communication ability. Using the inverted tent map feedback function in the higher dimensional chaotic regime, the performance is somewhat worse, as the channel noise interacts with the larger Lyapunov exponents, resulting in greater desynchronization. Still, the overall message is that synchronization of a semiconductor laser with optoelectronic feedback is structurally stable.

\section{DISCUSSION}

We have analyzed the chaotic oscillations of a semiconductor laser with optoelectronic feedback, as shown in Fig. 1. The use of the photodiode in the feedback loop was motivated by our inability to use the rapidly varying optical phase of laser systems in synchronization or communications applications [4]. Transforming the operational range of the feedback loop from op-

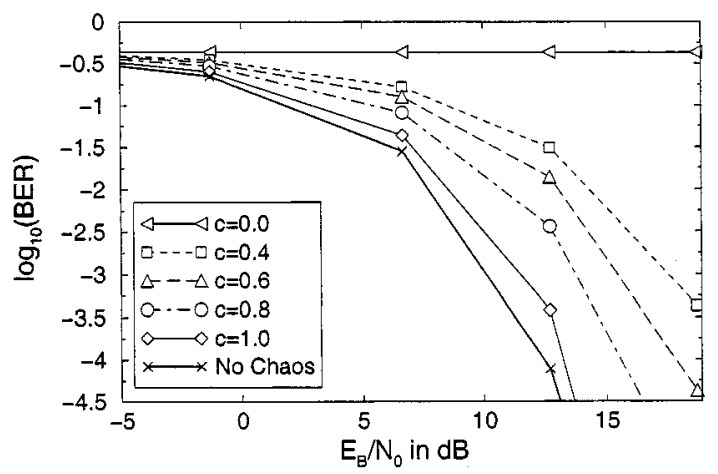

(a)

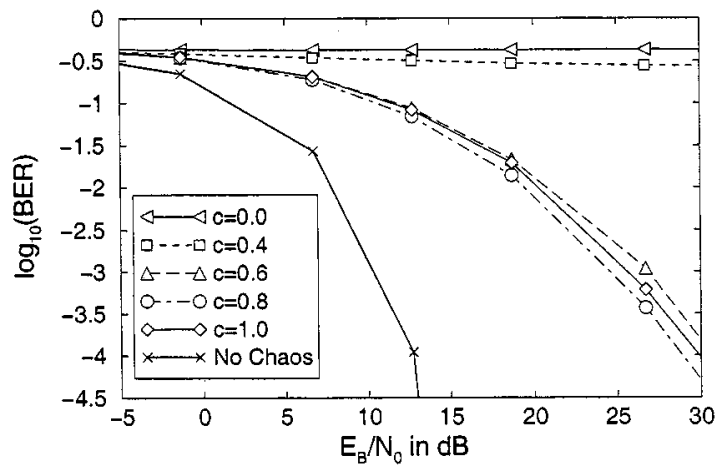

(b)

Fig. 15. BER versus effective SNR over a range of coupling strengths. (a) Linear feedback (thin lines). (b) Inverted tent map feedback (thin lines). Direct signaling (crosses) shown in both.

tical frequencies to high-speed electronics allows us to manipulate the feedback signal in an efficient and realistic fashion. We studied the idea of introducing a nonlinear function implemented electronically into the feedback loop, and demonstrated that it would produce high-dimensional chaotic oscillations. The feedback adds a time-delayed current $J_{F}\left(t-\tau_{R}\right)$, which we add to the laser bias current to close the feedback loop. $\tau_{R}$ is the propagation delay around the ring. While, in principle, the number of degrees of freedom in the time delay semiconductor laser system is infinite, in practice the number of active degrees of freedom depends on the magnitude of $\tau_{R}$ relative to the other time scales in the problem-see Table I for these. As a function of $\tau_{R}$, there is a bifurcation sequence as seen in Fig. 4 which reveals the richness of the structure when $J_{F}\left(t-\tau_{R}\right)$ is taken to be proportional to the intensity of the laser light $J_{F}\left(t-\tau_{R}\right)=\eta\left|A\left(t-\tau_{R}\right)\right|^{2}$. With the nonlinear, inverted tent map feedback considered in this paper, the bifurcation sequence is equally rich and the dimension of the chaotic oscillations larger at comparable $\tau_{R}$.

Synchronization of two similar lasers, one acting as a transmitter and sending a signal unidirectionally to the other acting as a receiver, is accomplished by injecting a fraction of the transmitter intensity $c\left|A_{T}(t)\right|^{2}$ added to a complementary fraction of the receiver intensity $(1-c)\left|A_{R}(t)\right|^{2}$ into the receiver laser through the injected current. The scheme for this is shown in Fig. 12 with $m(t)=1$. As a function of $0 \leq c \leq 1$, we have investigated the identity synchronization of two such lasers. For $c$ roughly 0.4 or larger, very accurate synchronization of the elec- 
tric field and carrier inversion in transmitter and receiver are accomplished both with and without spontaneous emission noise. Tang and Liu [16] have demonstrated synchronization of such systems.

No real semiconductor lasers are ever identical as our mathematical formulation would appear to require. To determine the sensitivity of our setup to parameter mismatch in real lasers, we evaluated a measure of synchronization, the time-averaged difference between transmitter and receiver intensities, as a function of mismatches in both intrinsic parameters of the lasers, such as the photon lifetime, and the carrier relaxation rate, and as a function of the feedback parameter $\xi$ and the feedback loop time $\tau_{R}$. For each parameter mismatch, the sensitivity of the synchronization was much larger at $c=0.4$ than at $c=1$, suggesting that $c=1$ is preferable in practice. Only the sensitivity to $\tau_{R}$ remained marked at $c \neq 1$, and this means that matching of the feedback delays will be required to use this kind of setup for communications based on the synchronization of the chaotic lasers.

Finally, we examined some communications strategies with and without channel noise. Channel noise is unlikely to be a major issue in optical fiber links, but in transmission though air, noise is likely to be important. Our evaluation of the BERs as a function of signal to noise in the channel summarized in Fig. 15 shows that quite acceptable performance can be achieved using the modulation/synchronization scheme we indicate in Fig. 12. The independence of the amplitude of the modulation for the synchronization error rate is an attractive feature of our method.

The work in this paper and a related paper on the experimental realization of these laser systems [16] prepares the way for further investigations of such systems; , in particular, the study of their ability to realistically work in a noisy environment and the analysis of multiuser methods using these coupled chaotic semiconductor lasers.

\section{REFERENCES}

[1] G. D. VanWiggeren and R. Roy, "Communication with chaotic lasers," Science, vol. 279, pp. 1198-1200, Feb. 1998.

[2] H. D. I. Abarbanel and M. B. Kennel, "Synchronizing high-dimensional chaotic optical ring dynamics," Phys. Rev. Lett., vol. 80, pp. 3153-3156, Apr. 1998.

[3] G. D. Van Wiggeren and R. Roy, "Optical communication with chaotic waveforms," Phys. Rev. Lett., vol. 81, pp. 3547-3550, Oct. 1998.

[4] H. D. I. Abarbanel, M. B. Kennel, M. Buhl, and C. Lewis, "Chaotic dynamics in erbium-doped fiber ring lasers," Phys. Rev. A, vol. 60, pp. 2360-2374, Sept. 1999

[5] T. B. Simpson, J. M. Liu, A. Gavrielides, V. Kovanis, and P. M. Alsing, "Period-doubling route to chaos in a semiconductor laser subject to optical injection," Appl. Phys. Lett., vol. 64, pp. 3539-3541, June 1994.

[6] S. Tang and J. M. Liu, "Chaotic pulsing and quasiperiodic route to chaos in a semiconductor laser with delayed optoelectronic feedback," IEEE J. Quantum Electron., vol. 37, pp. 329-336, Mar. 2001.

[7] E. V. Grigorieva, H. Haken, and S. A. Kaschenko, "Theory of quasiperiodicity in model of lasers with delayed optoelectronic feedback," Opt. Commun., vol. 165, pp. 279-292, July 1999.

[8] D. Pieroux, T. Erneux, T. Luzyanina, and K. Engelborghs, "Interacting pairs of periodic solutions lead to tori in lasers subject to delayed feedback," Phys. Rev. E, vol. 63, 036211 (11 pages), Feb. 2001.
[9] N. F. Rulkov, M. M. Sushchik, L. Tsimring, and H. D. I. Abarbanel, "Generalized synchronization of chaos in directionally coupled chaotic systems," Phys. Rev. E, vol. 51, pp. 980-994, Feb. 1995.

[10] D. Y. Tang, R. Dykstra, M. W. Hamilton, and N. R. Heckenberg, "Stages of chaotic synchronization," Chaos, vol. 8, pp. 697-701, Sept. 1998.

[11] _ - "Observation of generalized synchronization of chaos in a driven chaotic system," Phys. Rev. E, vol. 57, pp. 5247-5251, May 1998.

[12] R. M. Gagliardi and S. Karp, Optical Communications. New York: Wiley, 1976.

[13] C. C. Chen and K. Yao, "Stochastic-calculus-based numerical evaluation and performance analysis of chaotic communication systems," IEEE Trans. Circuits Syst. I, vol. 47, pp. 1663-1672, Dec. 2000.

[14] _ "Numerical evaluation of error probabilities of self-synchronizing chaotic communications," IEEE Commun. Lett., vol. 4, pp. 37-39, Feb. 2000.

[15] G. Denk and S. Schaffler, "Adams methods for the efficient solution of stochastic differential equations with additive noise," Computing, vol. 59, pp. 153-161, 1997.

[16] S. Tang and J. M. Liu, "Synchronization of high-frequency chaotic optical pulses," Opt. Lett., vol. 26, pp. 596-598, 2001.

Henry D. I. Abarbanel, photograph and biography not available at the time of publication.

Matthew B. Kennel was born in Santa Monica, CA, in 1968. He received the B.A. degree in physics from Princeton University, Princeton, NJ, in 1989, and the Ph.D. degree in physics from the University of California at San Diego (UCSD) in 1995.

After a postdoctoral fellowship at the Oak Ridge National Laboratory, Department of Energy, he joined the Institute for Nonlinear Science at UCSD as an Assistant Research Scientist. His research interests are in chaotic communication and the application of information theoretical algorithms to observed nonlinear dynamics.

Lucas Illing was born in Halle, Germany, in 1973. He received the Vordiplom from Humboldt University, Berlin, Germany, in 1996. He is currently working toward the Ph.D. degree in physics at the University of California at San Diego (UCSD).

In 1997, he joined the Institute for Nonlinear Science, UCSD. His research is primarily concerned with chaos synchronization and communication using chaos.

S. Tang received the B.S. and M.S. degrees in electronics from Peking University, Peking, China, in 1992 and 1997, respectively. She is currently working toward the Ph.D. degree in electrical engineering at the University of California, Los Angeles.

Her current research interests include optical communication systems, semiconductor lasers and nonlinear optics.

H. F. Chen, photograph and biography not available at the time of publication.

J. M. Liu, photograph and biography not available at the time of publication. 